\title{
THE USE OF THE TWO-HANDED COLLABORATIVE ROBOT IN NON-COLLABORATIVE APPLICATION
}

\author{
Michal VocetKa*, JiŘí Suder, Daniel Huczala \\ VSB - Technical University of Ostrava, Faculty of Mechanical Engineering, Department of Robotics, 17. \\ listopadu 2172/15, 70800 Ostrava - Poruba, Czech Republic \\ * corresponding author: michal.vocetka@vsb.cz
}

\begin{abstract}
The article deals with possibilities of using of a two-handed collaborative robot in automated production. The introductory part of this paper is about robot manufacturers' proposed ways of use of collaborative robots and a consideration of correctness of this stance. In this matter, an alternative point of view is proposed and tested, where a collaborative robot does not cooperate with a worker but replaces him/her completely. The main part of the study focuses on a specific installation of the YuMi collaborative robot into an already existing production line of a leading Czech supplier in the automotive industry. This real application is verified in simulations with an alternative solution consisting of two traditional industrial robots ABB IRB 120 instead. These data are evaluated and the advantages of deploying the collaborative robot and the industrial robots in the specific assembly application are compared. Economic return and productivity in high production cycle applications are considered. The article then describes the difficulties caused by the low load capacity of the YuMi collaborative robot and an alternative approach using the FEM methodology and topology optimization in the robot grip jaws design.
\end{abstract}

KEYworDS: Collaborative robot, YuMi, industrial robot, industrial automation, semi-collaborativ.

\section{INTRODUCTION}

In 2008, a Danish company Universal Robots had launched the first collaborative robot (cobot) that has a worldwide sales success and changed the established rules of the market $[1$. The small UR3 2 is simple to operate and thanks to its favourable price and collaborative principle, it is also available to customers who would not otherwise consider traditional industrial robotics. Most of the world leading robot manufacturers have sensed the potential of this idea and come up with their own designs and solutions.

The UR concept is a concept of the traditional, sixaxis kinematic structure, but with less robust tubular construction. The robots have an excellent load-toweight ratio, in the case of the UR3, it is $3: 11 \mathrm{~kg}$. It is very easy to control and install the cobot, after putting it out of the box, it is possible to put it in service within an hour. These cobots are very convenient for simple applications.

Taiwan enterprise Techman produces conceptually the same robots but improved by adding a camera system installed on its fifth axis [3]. The system can then, to some extent, evaluate the work environment by itself.

Fanuc has created the strongest cobot so far. Their CR-35iA has a load capacity of $35 \mathrm{~kg}$ [4]. According to many, such a weight defies collaboration, because dropping an object of this weight could easily cause pain and injury, which collaborativity does not allow. However, Fanuc offers this system to applications where it works as a hand-guided manipulator, the operator holds a control located on the upper arm and guides the manipulator where needed.

ABB has come up with a completely new concept. The cobot YuMI is supposed to work with person directly by being seated at one desk in front of the operator and work simultaneously, in the same work space and on the same task. The Cobot is equipped with two seven-axis arms (most robots and cobots have a six-axis arm). These arms are set up in a common body with a built-in controller. A collaborative tool, the smartgripper, is included with the YuMi cobot. The basic configuration includes an electric gripper with a clamping force of up to $20 \mathrm{~N}$, the gripper can be fitted with one or two suction cups and possibly a camera. [5]

This Cognex AE3 industrial camera has a resolution of 1.3 Mpx and an integrated LED for illumination. The standard robot control system is upgraded with the integrated vision module to control it. Also, it is possible to control the smart gripper camera through an InSight software, provided by Cognex [6]. This solution is designed for industrial-use, but camera guidance is time consuming.

The principle of cooperativity has also caught attention of people outside of the industrial automation, this type of automation is now widely supported by various government grants, large enterprises have ordered deploying cobots in applications for which it often does not seem to fit. The reason is the fear and apprehension that industrial automation, and especially robotization, will exclude people from pro- 

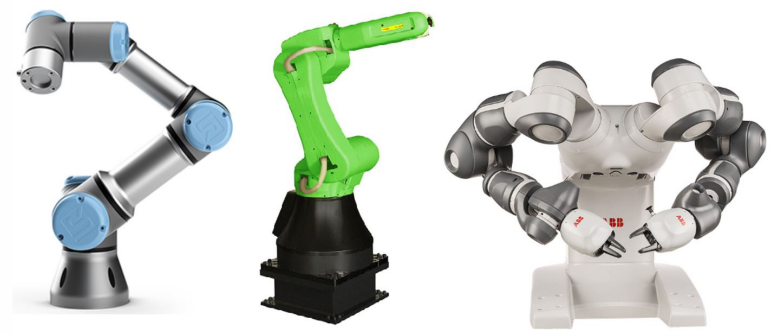

Figure 1. UR3, CR-35iA and YuMi cobots.

duction processes and increase unemployment, and so the cobotics is perceived as a compromise.

Collaborative robotics has many advantages, but it has some limitations too making it in most manufacturing processes so disadvantageous that it cannot stand in comparison with traditional industrial robotics. These limitations result from the safety functions and operation of these manipulators, which do not have to be installed behind the protective fence, optical barriers, zone scanners and are generally considered to be safe (which applies only to the manipulator, not to its tool and surrounding technology). The disadvantage is that the robots have a small load capacity, and, above all, are slow. It is not physically possible to create a fast robot with a kilogram load capacity that will be safe at the same time. In case of a collision with a human, more energy than would be acceptable could be transmitted. Moreover, according to the European legislation, any contact with human head and neck at any speed is completely unacceptable [7, 8]. Therefore, the question how to use cobots in practice arises 9 -13].

\section{A sPeCific YuMi application}

In the specific case described below, the choice of YuMi cobot is more appropriate in all aspects than the other considered options with traditional industrial robots. The task is to install a motor, a centrifugal clutch, an upper and a lower housing. The process is now carried out by a human and proceeds as follows: The operator puts both plastic housings into the press; the motor and the centrifugal clutch are inserted into the press as well. The automatic press gradually compresses all parts. The finished part is taken out from the press by the operator and placed in a technological palette. Due to the lack of workers, however, there is a risk of production interruption and the manufacturer is therefore trying to fully automate these simpler processes. However, the production must not be disturbed, so the installation of any new equipment must take place very quickly, and, if necessary (in the case of a robot malfunction), it must be possible to disconnect the robot immediately and to carry out the production by a human operator. It is also not possible to fence the workplace because of a lack of space. The last limiting parameter is the production cycle, which is set at $12 \mathrm{~s}$. These requirements directly

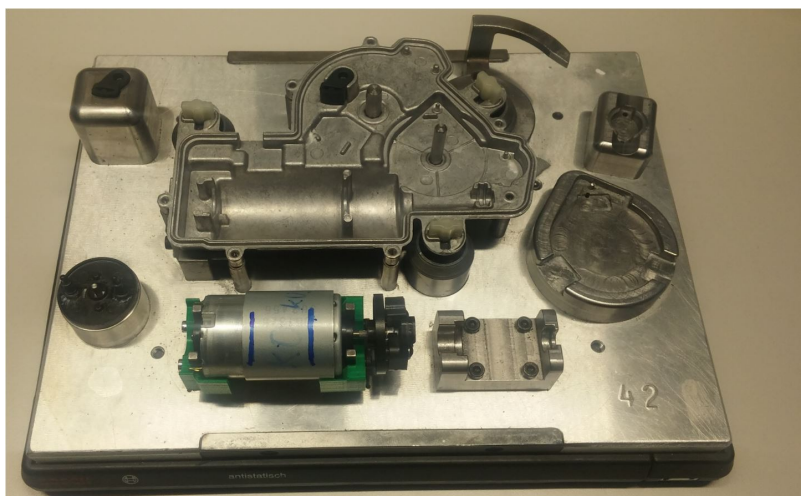

Figure 2. Bosch technological pallet.

lead to the use of a robot that works in a similar way to a human being. In addition, the installation of the YuMi cobot is also suitable because of the limited working space on the pallet and the presses. Indeed, YuMi has seven-axis arms that are much smaller in cross-section than the smallest industrial ABB robots. A pair of these industrial robots would not fit into the target space at the same time, the robots could not work simultaneously, which results in a longer duty cycle [14, 15.

The Fig. 2 above depicts the Bosch technology palette, the clutch is in the left corner, and the completed motor is placed in the green socket.

The proposed workstation consists of a press that is placed behind the pallet conveyor, a bowl feeder for housing supply and a robot on a docking station. The press had to be adapted for automation purposes, the pressing of the housings takes place separately and is provided by pneumatic cylinders with a diameter of $20 \mathrm{~mm}$. The pneumatic manipulator, whose individual drives only move between the end positions, solves the transfer of housings from the draw-off plate.

This solution is suitable with respect to price and adjusting the machine. The pressing plate can be rotated by 180 degrees. Pressing takes place on the front side while the back side is filled with the new set of housings by a pneumatic manipulator. The clutch is pressed on the motor by a pneumatic cylinder with a diameter of $80 \mathrm{~mm}$, the motor is locked by an angular gripper during pressing to avoid misalignment.

In Fig. 3 the Yumi robot is in the foreground and the manual assembly press is on its right side. Opposite the robot, the automatic press is mounted - it works only when the robot is connected and operational. The housings are delivered by the bowl feeder installed in the background.

The robot duty cycle differs slightly from the manual assembly. Left arm realizes pressing of the housings, right arm assembles the clutch to the motor. The whole process is tuned, so there is no delay caused by the arms waiting for each other.

Deploying and testing of a new technology on an already functioning production line that cannot be stopped for a longer time is difficult. For this reason, 


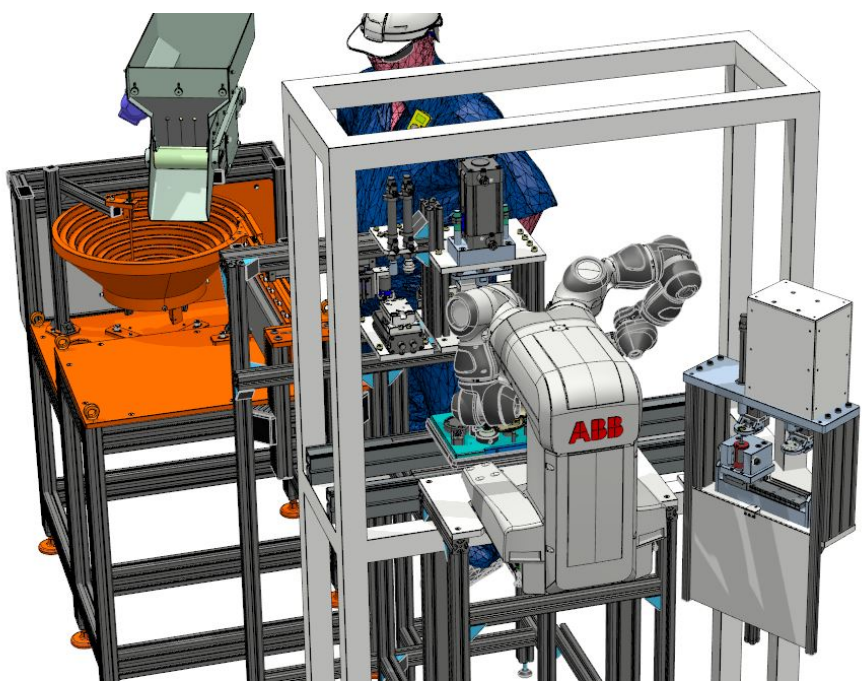

FiguRE 3. Assembly line workstation.

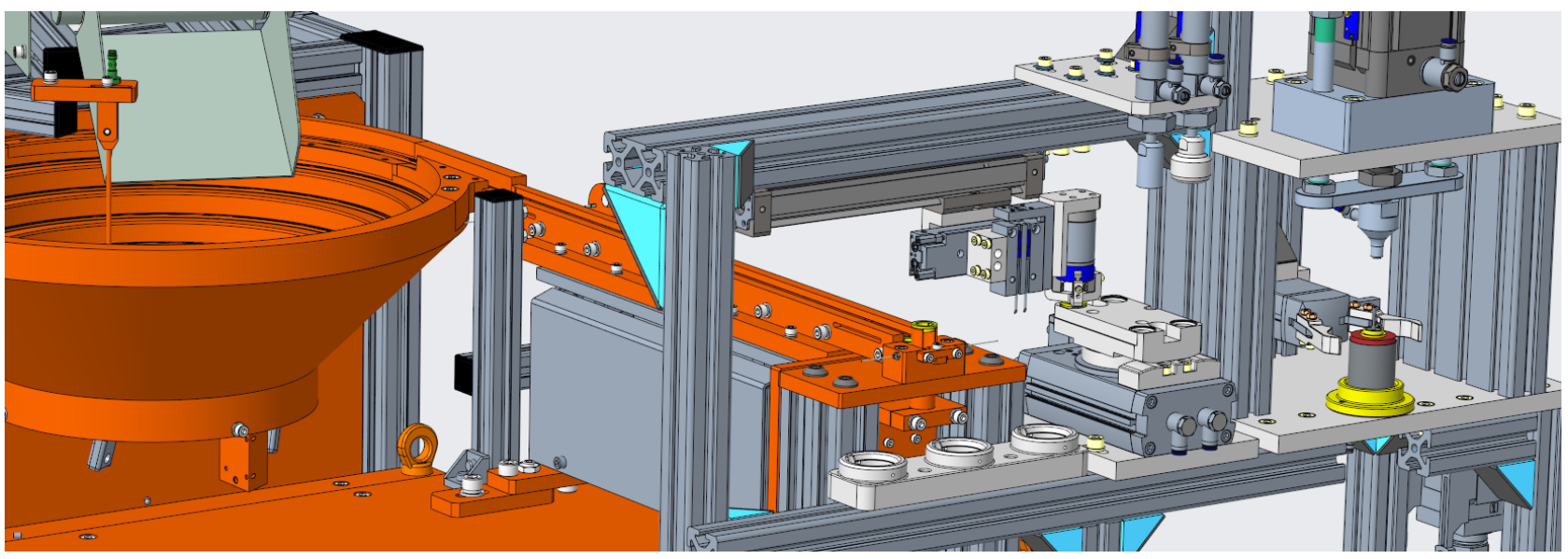

Figure 4. Automatic press detail.

the robot is placed on a detachable docking station. If this new technology does not work properly, then it can be easily disconnected, and another robot or line operator could continue the work manually instead. The workplace remains unchanged from the worker's point of view, but the production process will be stabilized after debugging, as the line performance is proportional to the performance of the slowest operator, and many tasks are performed at this station in a short time.

\section{LOW LOAD CAPACITY PROBLEMS}

However, the load capacity of the YuMi robot is problematic. It is set by the manufacturer at a maximum of $500 \mathrm{~g}$ as shown in Fig. 6. This weight must not exceed the sum of the weight of the object of manipulation and the end-effector. With these values, the maximum permissible weight of a one gripper jaw is $12 \mathrm{~g}$ when the smart gripper is used. If it is made of duralumin and the functional surface is coated with rubber, this weight can be achieved, but the robot will work on its limits. An alternative is a design of a custom end-effector with a pneumatic gripper, which

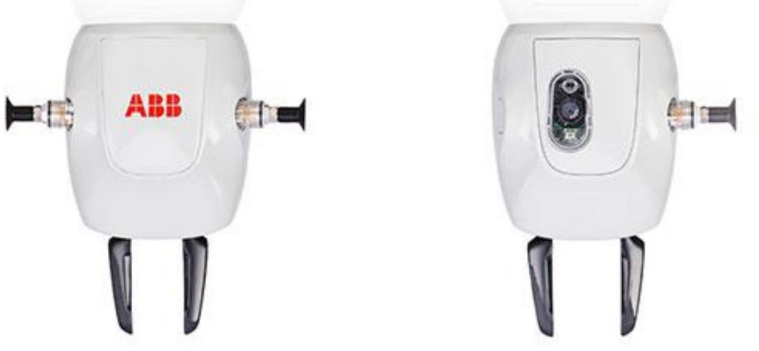

FiguRE 5. ABB Smart Gripper.

will have a quadruple clamping force, which is very desirable, total price per the tool will be lower, but it will not meet the principles of cooperativity.

The design of the jaw for the collaborative gripper was created based on the results of the topology optimization. In order to minimize the weight, this method seems to be the most appropriate.

The initial testing revealed that the jaw contact surface must copy the radius of the motor body on the largest possible area, otherwise there is a risk of displacement or rotation of the motor in the jaws. 


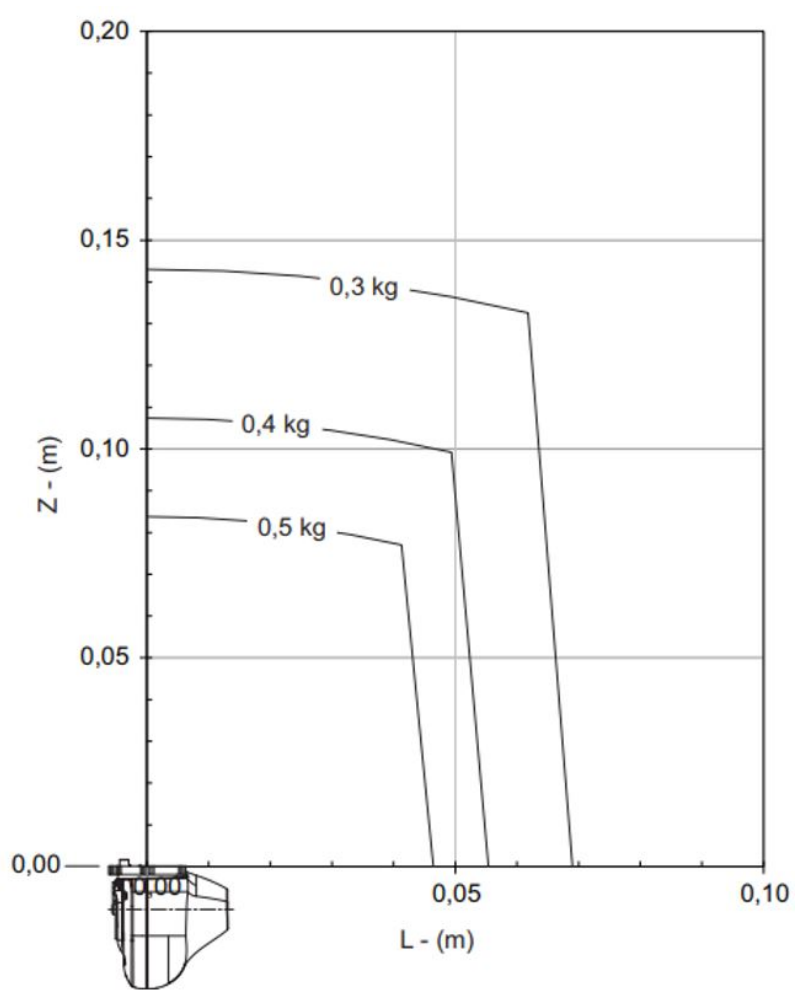

Figure 6. YuMi load diagram.

\begin{tabular}{ccc}
\hline material & mass $[\mathrm{g}]$ & tension $[\mathrm{MPa}]$ \\
\hline PC & 11.5 & $10-20$ \\
AL6061 & 23.9 & \\
\hline
\end{tabular}

TABle 1. Comparison of FEM analysis results.

In addition, this surface must be coated with a soft coating to enhance adhesion.

The original design of the jaw, which would be printed on a $3 \mathrm{D}$ polycarbonate printer, corresponds to a strength and weight limits, but the lifetime of such a jaw would be short, the $3 \mathrm{D}$ printed part would not withstand the cyclic loading. However, in the case of the same design but using higher quality aluminium instead, the weight would rise above the acceptable limit, as it is shown in Table 1

The principle of topology optimization is to exclude all the mass, which does not carry any load and is therefore useless, from the optimized part. The FEM analysis has shown that this part has a potential to be topologically optimized. In the case of the aluminium, the optimization results in half the weight, at roughly doubling the stress values, but this is still acceptable. Thus, the optimized part will be modified to reduce the production costs. However, it can be considered as sufficient at this phase.

\section{INDUSTRIAL ROBOT SOLUTION}

The possible solution with a pair of traditional six-axis robots is also functional, but in comparison with the YuMi cobot, it is slower by two seconds.

\begin{tabular}{ccc}
\hline material & mass $[\mathrm{g}]$ & tension $[\mathrm{MPa}]$ \\
\hline $\mathrm{PC}$ & 5.6 & $25-40$ \\
AL6061 & 12 & \\
\hline
\end{tabular}

TABle 2. Comparison of FEM analysis results of topologically analyzed part.

\begin{tabular}{lcc}
\hline Parameter & YuMi & $2 \times$ IRB120 \\
\hline investment & $\mathbf{6 0 ~ 0 0 0 ~} \mathbf{~}$ & $66500 €$ \\
tact & $\mathbf{1 0 . 5} \mathbf{~ s}$ & $12.8 \mathrm{~s}$ \\
modularity & Yes & No \\
fenced & No & Yes \\
power excess & No & Yes \\
\hline
\end{tabular}

TABle 3. Comparison of FEM analysis results of topologically analyzed part.

The industrial solution simulation contemplates a pair of ABB IRB120 robots. These small six - axis industrial robots could reach a speed of $6.2 \mathrm{~m} / \mathrm{s}$ and acceleration of $28 \mathrm{~m} / \mathrm{s}^{2}$ with a load capacity of $3 \mathrm{~kg}$. [16]

Robots would be faster, if they were hung, but this option is not possible. Even though the robot is generally much faster, it does not reach higher speeds on such a short trajectory, the mightiness of its arms causes additional delays when one robot waits for another.

All the simulations were performed in ABB RobotStudio software. It is probably the most accurate software to simulate ABB robots, according to a fact that a detailed robot specification and performance is confidential, considered as a brand's know-how.

The last and probably the most serious parameter is the price, where the solution will be more expensive by an estimated $€ 6,500$. Based on these parameters, the cobot YuMi seems to be more appropriate, for this specific case.

\section{Results}

The total cost of the automation is estimated at about $60,000 €$. Considering that the line operates in twoshift operation and so two operators will be spared, the return is estimated to be about three years. The more important fact is that the production will not be hampered by a lack of personnel, the robotized station is three seconds faster than a human operator, so there may be a small increase in production.

The comparison of cobot and industrial robots has shown that a cobot is more appropriate in this case. The parameters are compared in Table 3. The key parameters in the decision making are the price and modularity, i.e. whether it is necessary to fence the station and whether it is possible to quickly replace the cobot with another cobot or a human.

This comparison can be surprising, but it is valid only for this specific application. In other applications, 

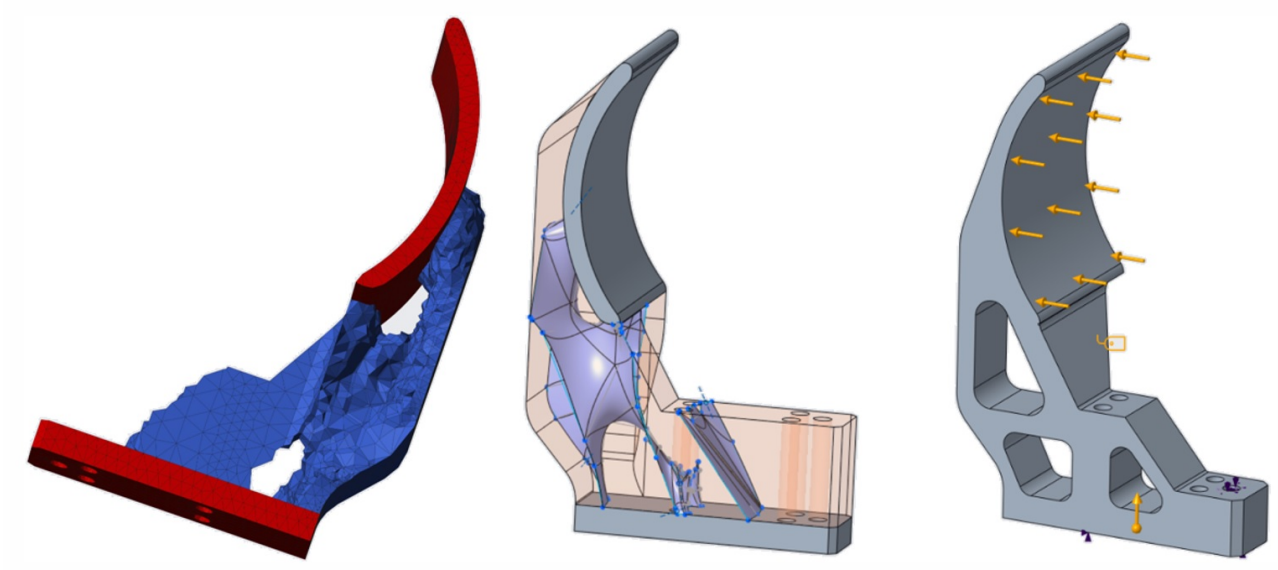

Figure 7. Topology optimization process.
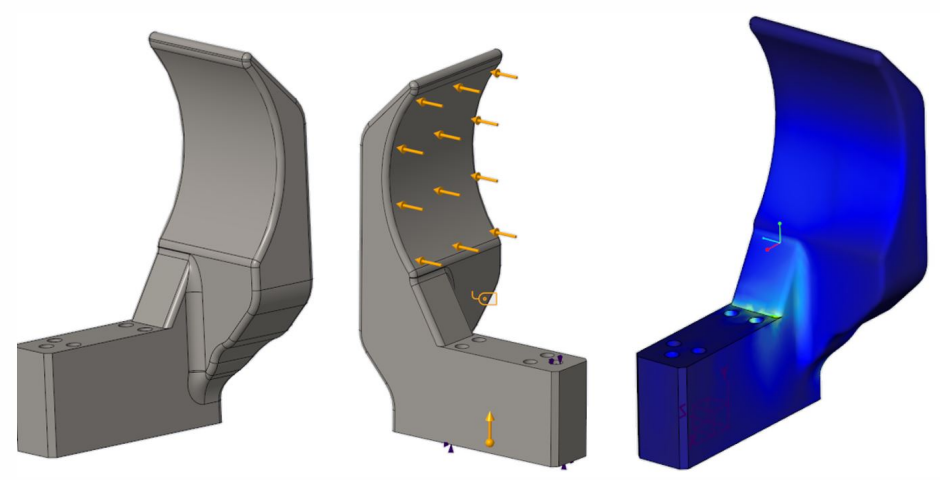

Figure 8. FEM analysis.

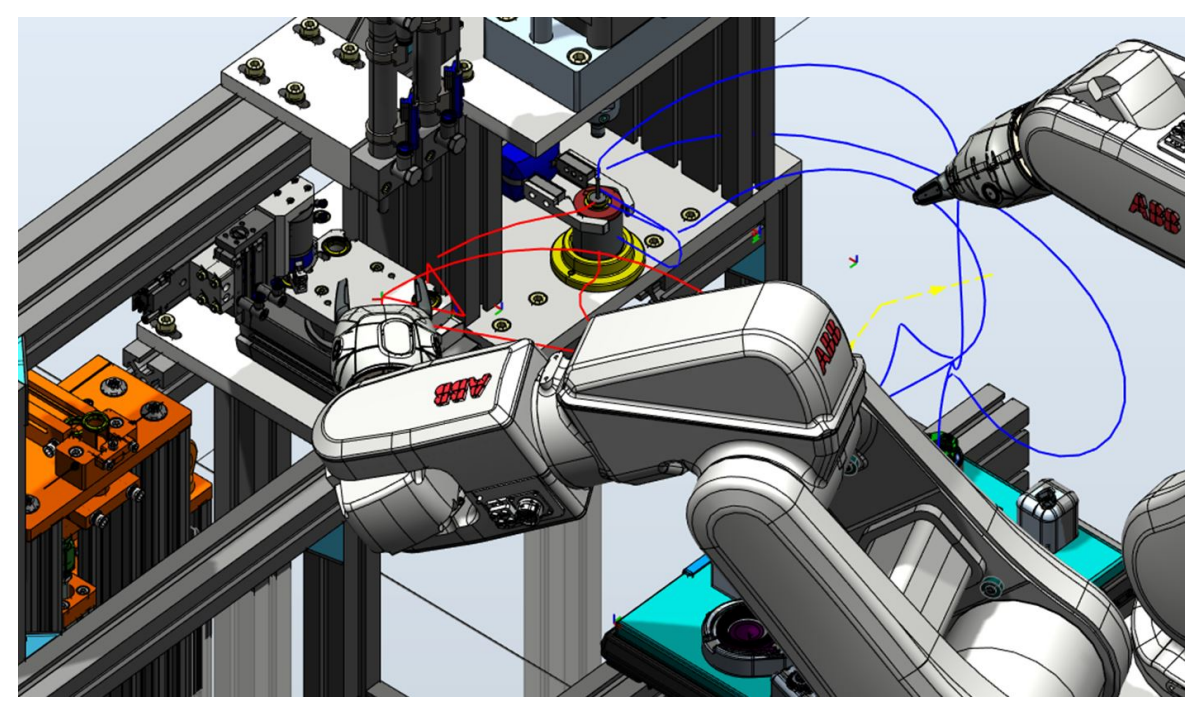

Figure 9. A pair of IRB120. 
YuMi would probably not be able to withstand the comparison with industrial robots.

\section{Conclusion}

It is known that cobots are very suitable for applications with a long production cycle. In industrial applications, there are many cases where the cobot is installed in a manner of the industrial robot, behind the fence, and it works in a non-collaborative mode. This approach may be advantageous in terms of the cost of the manipulator.

The cobots are very useful tools for programming and robot-control education. In those applications, there is no need for high speeds and the cobot can move unloaded. Due to the safe operation of the manipulator itself, which does not need safety peripherals for its function, this is a suitable solution for technical schools and universities or training centres that offer this type of education.

In this specific case, surprisingly, a situation where cobot deployment is more useful than a robot, has been achieved.

A standard collaboration would not by possible, due to the surrounding technology, such as presses, conveyor etc.

A traditional industrial robot solution is applicable, but requires more floor space and needs to be fenced, so there is no room for modularity.

The idea of using a semi-collaborative installation would be interesting. The YuMi robot works as a standard industrial manipulator, using maximum speed and acceleration, however, its body covers the workspace and YuMi parameters are not that dangerous. Protection in a way of light curtain, whose disruption will result in a stop of all presses and cobot as well, is acceptable. This safety solution, together with small docking station for a small cobot saves the floor space, workstation could be "opened" and so robot could be easily and quickly replaced, even by a human worker.

This idea is economical especially in case of lowvolume production, or production of several product variants. If a variant doesn't need, for example, a technological operation provided by the cobot, that cobot could be used on another production line in another manufacturing process, as is needed.

As the next steps of research, we would like to work on adaptive jaws. This kind of jaws is already available on the market [17], but we believe that for cylindrical object of manipulation, a better FinRay jaw design could be invented.

\section{ACKNOWLEDGEMENTS}

This work was supported by the European Regional Development Fund in the Research Centre of Advanced Mechatronic Systems project, project number CZ.02.1.01/0.0/0.0/16_019/0000867 within the Operational Programme Research, Development and Education and project VEGA $1 / 0355 / 18$ The use of experimental methods of mechanics for refinement and verification of numerical models of mechanical systems with a focus on composite materials. This article has been also elaborated under support of the Specific Research Project SP2019/69 and financed by the Ministry of Education, Youth and Sports of the Czech Republic.

\section{REFERENCES}

[1] Universal robots. https://en.wikipedia.org/wiki/Universal_Robots 2019. Accessed: 19 March 2019.

[2] Universal robots. https://www. universal-robots.com/cs/, 2019. Accessed: 19 March 2019.

[3] Techman robots. http://tm-robot.com/TM5.php 2019. Accessed: 19 March 2019.

[4] FANUC. https :

//www.fanuc.eu/cz/en/robots/robot-filter-page/ collaborative-robots/collaborative-cr35ia, 2019. Accessed: 19 March 2019.

[5] ABB YuMi - IRB14000. https://new.abb.com/products/robotics/cs/ prumyslove-roboty/yumi?utm_source=google\&utm_ campaign=SE_Roboty_Roboty\&utm_medium=cpc\&utm_ term=Roboty\&utm_content=Robot-YUMI\&gclid= EAIaIQobChMItqTrrJ-04QIVQZztCh06BAyrEAAYASAAEgJVovD_ BwE, 2019. Accessed: 19 March 2019.

[6] Product manual - IRB 14000 gripper: Document ID: 3HAC054949-001. https://abb.sluzba.cz/Pages/ Public/IRC5UserDocumentationRW6/en/3HAC054949\% 20PM $\% 20$ IRB $\% 2014000 \% 20$ Gripper-en.pdf, 2018.

Accessed: 12 February 2020.

[7] ISO 10218 - Robots for Industrial Environments Safety Requirements-Part 1: Robot. Standard, International Organization for Standardization, Geneva, Switzerland, 2006. This version is no longer in effect.

[8] ISO/TS 15066 - Robots and robotic devices Collaborative robots. Standard, International Organization for Standardization, Geneva, Switzerland, 2016.

[9] V. Villani, F. Pini, F. Leali, C. Secchi. Survey on human-robot collaboration in industrial settings: Safety, intuitive interfaces and applications. Mechatronics $\mathbf{5 5}: 248-266,2018$. DOI:10.1016/j.mechatronics.2018.02.009.

[10] V. Villani, F. Pini, F. Leali, et al. Survey on human-robot interaction for robot programming in industrial applications. IFAC-PapersOnLine 51(11):66 71, 2018. 16th IFAC Symposium on Information Control Problems in Manufacturing INCOM 2018, DOI:10.1016/j.ifacol.2018.08.236

[11] G. Michalos, S. Makris, P. Tsarouchi, et al. Design considerations for safe human-robot collaborative workplaces. Procedia CIRP 37:248 - 253, 2015. CIRPe 2015 - Understanding the life cycle implications of manufacturing, DOI:10.1016/j.procir.2015.08.014.

[12] J. Fryman, B. Matthias. Safety of industrial robots: From conventional to collaborative applications. In German Conf. Robot., ROBOTIK, pp. $1-5$. Munich, Germany, 2012. 
[13] I. Maurtua, A. Ibarguren, J. Kildal, et al. Human-robot collaboration in industrial applications: Safety, interaction and trust. International Journal of Advanced Robotic Systems 14(4), 2017. DOI:10.1177/1729881417716010

[14] A. M. Zanchettin, N. M. Ceriani, P. Rocco, et al. Safety in human-robot collaborative manufacturing environments: Metrics and control. IEEE Transactions on Automation Science and Engineering 13(2):882 893, 2016. DOI:10.1109/TASE.2015.2412256

[15] H. Ding, J. Heyn, B. Matthias, H. Staab. Structured collaborative behavior of industrial robots in mixed human-robot environments. In IEEE International Conference on Automation Science and Engineering, CASE. 2013.
[16] Product specification: IRB 120. https://library.e.abb.com/public/ 7139d7f4f 2cb4d0da9b7fac6541e91d1/3HAC035960\% 20PS\%20IRB\%20120-en.pdf Accessed: 11 February 2020 .

[17] FESTO. Adaptive gripper fingers DHAS. https://www.festo.com/cat/cs_cz/data/doc_engb/ PDF/EN/DHAS_EN.PDF, 2017. Accessed: 27 February 2020. 